\title{
Article \\ Role of MiR-325-3p in the Regulation of CFL2 and Myogenic Differentiation of C2C12 Myoblasts
}

\author{
Mai Thi Nguyen ${ }^{1}$ (D) and Wan Lee ${ }^{1,2, *(D)}$ \\ 1 Department of Biochemistry, Dongguk University College of Medicine, 123 Dongdae-ro, \\ Gyeongju 38066, Korea; nguyenmainhp@gmail.com \\ 2 Channelopathy Research Center, Dongguk University College of Medicine, 32 Dongguk-ro, \\ Goyang 10326, Korea \\ * Correspondence: wanlee@dongguk.ac.kr; Tel.: +82-54-770-2409
}

Citation: Nguyen, M.T.; Lee, W. Role of MiR-325-3p in the Regulation of CFL2 and Myogenic Differentiation of C2C12 Myoblasts. Cells 2021, 10, 2725. https://doi.org/10.3390/ cells10102725

Academic Editor: Yuko

Miyagoe-Suzuki

Received: 18 August 2021

Accepted: 27 September 2021

Published: 12 October 2021

Publisher's Note: MDPI stays neutral with regard to jurisdictional claims in published maps and institutional affiliations.

Copyright: (c) 2021 by the authors. Licensee MDPI, Basel, Switzerland. This article is an open access article distributed under the terms and conditions of the Creative Commons Attribution (CC BY) license (https:// creativecommons.org/licenses/by/ $4.0 /)$.

\begin{abstract}
Skeletal myogenesis is required to maintain muscle mass and integrity, and impaired myogenesis is causally linked to the etiology of muscle wasting. Recently, it was shown that excessive uptake of saturated fatty acids (SFA) plays a significant role in the pathogenesis of muscle wasting. Although microRNA (miRNA) is implicated in the regulation of myogenesis, the molecular mechanism whereby SFA-induced miRNAs impair myogenic differentiation remains largely unknown. Here, we investigated the regulatory roles of miR-325-3p on CFL2 expression and myogenic differentiation in C2C12 myoblasts. PA impeded myogenic differentiation, concomitantly suppressed CFL2 and induced miR-325-3p. Dual-luciferase analysis revealed that miR-325-3p directly targets the $3^{\prime}$ UTR of CFL2, thereby suppressing the expression of CFL2, a crucial factor for actin dynamics. Transfection with miR-325-3p mimic resulted in the accumulation of actin filaments (F-actin) and nuclear Yes-associated protein (YAP) in myoblasts and promoted myoblast proliferation and cell cycle progression. Consequently, miR-325-3p mimic significantly attenuated the expressions of myogenic factors and thereby impaired the myogenic differentiation of myoblasts. The roles of miR-325-3p on CFL2 expression, F-actin modulation, and myogenic differentiation suggest a novel miRNA-mediated regulatory mechanism of myogenesis and PA-inducible miR-325-3p may be a critical mediator between obesity and muscle wasting.
\end{abstract}

Keywords: microRNA; miR-325-3p; CFL2; palmitic acid; proliferation; differentiation

\section{Introduction}

Skeletal muscle is essential for proper physical and systemic homeostasis, including locomotion, metabolism, and respiration [1]. The maintenance of skeletal muscle mass and integrity is regulated by myogenesis, which involves satellite cell activation, myoblast differentiation, and myotube formation [2]. Accordingly, dysregulation of myogenesis induces muscle wasting diseases, including sarcopenia and cachexia, and increases the risks of frailty, morbidity, and mortality [2,3]. Numerous studies have indicated that muscle wasting is derived from diverse conditions that impede myogenesis, such as oxidative stress, mitochondrial dysfunction, and senescence [3,4]. Recently, it was suggested that obesity exacerbates the detrimental consequences of muscle wasting and leads to sarcopenic obesity $[5,6]$. Excess uptake of saturated fatty acids (SFA) increases intramuscular fat infiltration and frequently provokes muscle wasting $[7,8]$. Furthermore, many studies have shown that certain microRNAs (miRNAs) dysregulated by SFA, and obesity are associated with muscle wasting $[9,10]$.

MiRNAs are a class of short non-coding RNAs and play essential roles in a broad range of biological processes by targeting the $3^{\prime} \mathrm{UTR}$ regions of target mRNAs [11,12]. For this reason, aberrant miRNA expression is intimately associated with the pathogeneses of numerous diseases, including cancer, cardiovascular diseases, and metabolic disorders [13]. 
Recently, many studies have focused on the regulatory roles of miRNAs in muscle homeostasis, muscle wasting, and other myopathies $[14,15]$. Accumulating evidence indicates that several miRNAs are involved in muscle wasting through their inhibitory effects on myogenesis $[9,16]$. Nevertheless, the molecular mechanism whereby SFA-induced miRNAs suppress myogenic differentiation remains largely unknown.

Actin remodeling, coordinated by actin-binding proteins, modulates the cytoskeletal dynamics necessary for myoblast proliferation and differentiation [17,18]. Cofilin 2 (CFL2) is a skeletal muscle-specific actin-binding protein and belongs to the actin-depolymerizing factor (ADF)/cofilin family $[19,20]$. CFL2 plays an essential role in actin remodeling by severing or depolymerizing filamentous actin (F-actin), which is involved in muscle development and maintenance $[19,20]$. In a mouse model, the functional ablation of CFL2 was associated with skeletal muscle wasting accompanied by F-actin accumulation [21]. In addition, CFL2 knockout disrupted sarcomere structure and integrity with enhanced actin polymerization [22]. Furthermore, CFL1-mediated actin remodeling has been shown to regulate cell proliferation associated with myogenic differentiation $[23,24]$. In a previous study, we found that CFL2 knockdown by siRNA promoted myoblast proliferation and consequently inhibited myogenic differentiation in C2C12 cells [25]. Although CFL2 is known to be crucial for skeletal myogenesis and maintenance, its regulation by miRNAs during myogenic differentiation has not been explored.

Here, we investigated the role of SFA-induced miRNA on myogenic differentiation. We found that miR-325-3p, markedly induced by palmitic acid (PA) in myoblasts, regulates CFL2 expression directly. We also showed that miR-325-3p plays a crucial role in cell proliferation, myogenic factors expressions, and differentiation in myoblasts. Our findings regarding the regulatory functions of miR-325-3p on myogenesis increase understanding of the mechanism of muscle wasting in the background of obesity and will provide a novel diagnostic and therapeutic target for muscle wasting and sarcopenic obesity.

\section{Materials and Methods}

\subsection{Cell Culture, Differentiation and PA Treatment}

C2C12 myoblasts, an immortalized murine muscle progenitor cell line (ATCC), were maintained in a growth medium (GM; Dulbecco's modified Eagle's medium (DMEM) containing $10 \%$ fetal bovine serum and $1 \%$ penicillin/streptomycin) (Gibco, Carlsbad, CA, USA) at $37^{\circ} \mathrm{C}$ in a $5 \% \mathrm{CO}_{2}$ humidified incubator. For the biochemical study, cells were seeded on 6-well plates (Thermo Fisher Scientific, Waltham, MA, USA) at a density of $1.3 \times 10^{5}$ cells/well in $2 \mathrm{~mL}$ of GM. After $24 \mathrm{~h}$, cells were transiently transfected with indicated oligonucleotides using Lipofectamine 2000 (Invitrogen, Waltham, MA, USA) according to the manufacturer's instructions. When cells reached $80-90 \%$ confluence, myoblasts were differentiated to myotubes by switching to a differentiation medium (DM; DMEM containing $2 \%$ dialyzed horse serum and $1 \%$ penicillin/streptomycin). When necessary, cells were treated with BSA-conjugated PA $(100 \mu \mathrm{M})$ for $24 \mathrm{~h}$ in GM before differentiation as described previously [26]. Unless otherwise stated, all reagents and materials were purchased from Sigma-Aldrich (St. Louis, MO, USA).

\subsection{Transfection of Oligonucleotides}

C2C12 myoblasts were transfected with scrambled control RNA (scRNA), CFL2 siRNA (siCFL2), miR-325-3p mimic, or antimiR-325-3p (an inhibitor of miR-325-3p; a 2'-O-methylmodified antisense oligonucleotide against mature miR-325-3p) from Genolution (Seoul, Korea) at final concentrations of $200 \mathrm{nM}$ in a GM using Lipofectamine 2000. The sequences of the oligonucleotides used for this study are listed in Table S1.

\subsection{RNA Extraction, $P C R$ and Quantitative Real-Time PCR ( $q R T-P C R$ )}

Total RNA of $\mathrm{C} 2 \mathrm{C} 12$ cells was extracted using a Qiazol reagent and miRNeasy Mini Kit (Qiagen, Hilden, Germany) $24 \mathrm{~h}$ after transfection. The quality and concentration of RNAs were assessed by gel electrophoresis and a UV-1700 PharmaSpec spectrophotometer 
(Shimadzu, Kyoto, Japan). The RNA was then reverse-transcribed using the miScript II RT Kit (Qiagen). To determine mRNA and miRNA expression levels, $q$ RT-PCR and RT-PCR were conducted using specified primers, SYBR Green I, and iTaq polymerase (Promega, Medison, WI, USA) in conjunction with a Light-Cycler 480 (Roche Applied Science, Penzberg, Germany). Details of the primers used for RT-PCR and $q$ RT-PCR and reaction conditions are described in Table S2. The $2^{-\Delta \Delta C t}$ method was used to calculate relative mRNA expressions, and results were normalized by U6 snRNA expression.

\subsection{Dual-Luciferase Assay}

A segment of the murine CFL2 3'UTR (358-nt long) containing the potential miR-325$3 p$ binding site (CFL2wt) was chemically synthesized by RT-PCR using specific primers listed in Table S2. The wild-type reporter construct was produced by subcloning CFL2wt into the pmirGLO vector (Promega) using SacI and XbaI sites. Mutation of the miR-325-3pbinding site (CFL2mut) was synthesized by PCR-based site-directed mutagenesis using overlapping oligonucleotides (Table S2). For dual-luciferase target validation assays, C2C12 cells were plated in a 12 -well plate at a density of $1 \times 10^{5}$ cells/well. After $24 \mathrm{~h}$, a pmirGLO vector containing CFL2wt or CFL2mut was co-transfected with scRNA or miR-325-3p mimic into cells using Lipofectamine 2000. The Dual-Luciferase Reporter Assay System 100 Kit (Abcam, Cambridge, UK) was used to determine luciferase activities $24 \mathrm{~h}$ after transfection as described recently [27].

\subsection{Immunoblot Analysis}

For protein preparation, C2C12 cells were collected by centrifugation and lysed using PBS containing 2\% Triton X-100 and 1\% phosphatase inhibitor cocktail II (Abcam) as previously described [28]. The NE-PER Nuclear and Cytoplasmic Extraction Reagents (Thermo Fisher Scientific) were used for nuclear and cytoplasmic protein fractionation. Protein concentrations were determined using the Bradford method, and then cell lysates were mixed with $2 X$ Laemmli buffer and boiled for $10 \mathrm{~min}$ at $100{ }^{\circ} \mathrm{C}$. Proteins were separated by SDS-PAGE at $20 \mu \mathrm{g}$ of protein/lane and transferred to nitrocellulose membranes (Amersham, Germany). The membranes were then blocked with 5\% skim milk in TTBS (0.5\% TBS-Tween 20 in PBS) for $1 \mathrm{~h}$ and incubated with specific primary antibodies at $4{ }^{\circ} \mathrm{C}$ overnight (Table S3). The membranes were washed with TTBS 5 times and incubated with a secondary antibody for $1 \mathrm{~h}$. Finally, protein bands were visualized using a Femto reagent (Thermo Fisher Scientific) with Fusion Solo (Vilber, Marne-la-Vallée, France), and their densities were determined by Evolution-Capt software (Vilber, Marne-la-Vallée, France). $\beta$-Actin protein levels were used for normalization.

\subsection{Immunofluorescence Analysis}

After transfection with the indicated oligonucleotides (Table S1), C2C12 cells were allowed to differentiate for five days. C2C12 myotubes were fixed and permeabilized using $4 \%$ paraformaldehyde and 0.3\% Triton X-100 in PBS. Cells were then blocked with 3\% BSA for $2 \mathrm{~h}$ at room temperature, incubated overnight with primary antibody (MyHC; 1:100 dilution), washed 3 times with PBS, and then incubated for an additional $1.5 \mathrm{~h}$ with secondary antibody (Alexa 488, Invitrogen; 1:200 dilution). In the case of F-actin staining, cells were incubated with $50 \mu \mathrm{g} / \mathrm{mL}$ FITC-conjugated phalloidin (P5282, Sigma, USA) for $40 \mathrm{~min}$ instead of MyHC antibody. Nuclei were counterstained with Hoechst 33342 (Invitrogen). All images were obtained using a fluorescence microscope (Leica Microsystems, Mannheim, Germany). Differentiation and fusion index were calculated as indicators of myotube formation. The differentiation index was defined as the number of nuclei expressed in MyHC-positive myotubes as a percentage of total nuclei. The fusion index was defined as the ratio of the total nuclei residing in myotubes with three or more nuclei. Myotube widths and MyHC-positive areas were analyzed using ImageJ software (ver 1.53, USA National Institutes of health, Bethesda, MA, USA). 


\subsection{Cell Proliferation Assays}

EdU assays were applied to evaluate cell proliferation using the Click-iT EdU Cell Proliferation Kit (Invitrogen). Cells were plated in chamber slides at a density of $3 \times 10^{4}$ cells/well. After $24 \mathrm{~h}$ of transfection, cells were treated for $4 \mathrm{~h}$ with EdU $(10 \mu \mathrm{M})$, fixed with formaldehyde (4\%) for $10 \mathrm{~min}$, and permeabilized with Triton X-100 (0.3\%) in PBS for $15 \mathrm{~min}$. Then, cells were incubated with $0.3 \mathrm{~mL}$ of Click-iT reaction cocktail for $20 \mathrm{~min}$, and nuclei were labeled for an additional $15 \mathrm{~min}$ with Hoechst 33342. Each image was taken with a Leica microscope. The total number of cells and the number of EdU-positive cells were counted in random pictures, and ImageJ software was used to calculate the percentages of EdUpositive cells and the total number of nuclei. All experiments were conducted at least three times using at least five randomly selected fields/experiment.

\subsection{Flow Cytometry}

C2C12 cells were collected $24 \mathrm{~h}$ after transfection, resuspended at a density of $1 \times 10^{6}$ cells, and centrifuged at $3000 \mathrm{rpm}$ for $5 \mathrm{~min}$ at $4{ }^{\circ} \mathrm{C}$. Pellets were washed three times with PBS, fixed with $70 \%$ ethanol overnight at $4{ }^{\circ} \mathrm{C}$, and treated with $500 \mu \mathrm{L}$ of Cell Cycle Kit solution (C03551, Beckman Coulter, Brea, CA, USA) for $20 \mathrm{~min}$ in the dark. Finally, cell cycle analyses were performed using a CytoFLEX (Beckman Coulter, Brea, CA, USA).

\subsection{Database and Statistical Analysis}

Using publicly accessible bioinformatics software (TargetScan: www.targetscan.org, Pictar: pictar.mdc-berlin.de), putative miR-325-3p binding sites on the 3UTR of CFL2 mRNA were identified. Results are presented as the means \pm standard errors of at least three independent experiments. The statistical analysis was performed using the Student's $t$-test for unpaired data.

\section{Results}

\subsection{PA Inhibited Myogenic Differentiation but Elevated miR-325-3p Expression}

Since CFL2 is necessary for myoblast differentiation [25], we investigated how CFL2 expression during differentiation is affected by SFA in myoblasts. C2C12 cells were treated with PA $(100 \mu \mathrm{M})$, the most abundant dietary SFA, for $24 \mathrm{~h}$ and then differentiated up to five days. Myoblast differentiation was then evaluated according to the myogenic factors expressions and myotube formation. PA-treatment remarkably decreased the MyHC-positive area and suppressed myoblast differentiation and fusion in C2C12 cells as determined by immunocytochemistry and quantitative image analysis (Figure 1A,B). In agreement with immunocytochemistry findings, PA significantly suppressed the levels of MyoD, MyoG, and MyHC (Figure 1C), indicating that PA dramatically impeded myogenic factors expressions and differentiation in $\mathrm{C} 2 \mathrm{C} 12$ myoblasts. Interestingly, under these conditions, the expression of CFL2 was significantly diminished by PA (Figure 1C,D). These results suggest that impaired myogenic differentiation by PA is associated with CFL2 suppression in myoblasts. Next, we investigated whether specific miRNAs upregulated by PA are implicated in CFL2 suppression in myoblasts. According to microarray results, the expression of miR-325-3p, which was predicted to target $3^{\prime}$ UTR of CFL2 with a high probability according to the miRNA target analysis using TargetScan and miRWalk, was upregulated $>1.5$-fold in PA-treated myoblasts (Supplementary Data). Therefore, miR-325$3 p$ was chosen for further investigation because it has been supposed to be associated with muscle atrophy and dystrophy $[29,30]$. The $q$ RT-PCR confirmed that PA raised miR-325$3 p$ expression in myoblasts by 3 -fold (Figure 1E). Collectively, PA was found to impair myogenic differentiation and suppress CFL2 expression but induce miR-325-3p expression in myoblasts. 
A

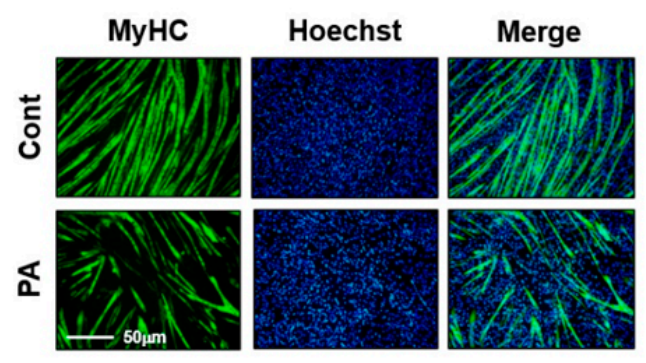

B
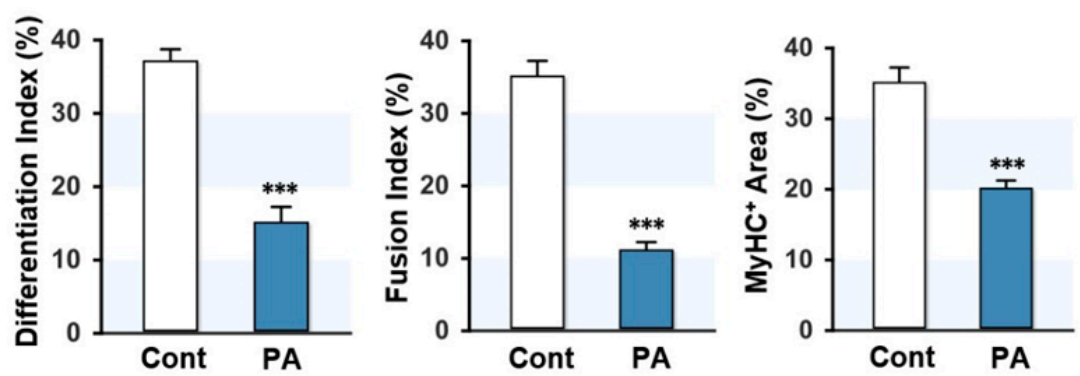

C

D

E
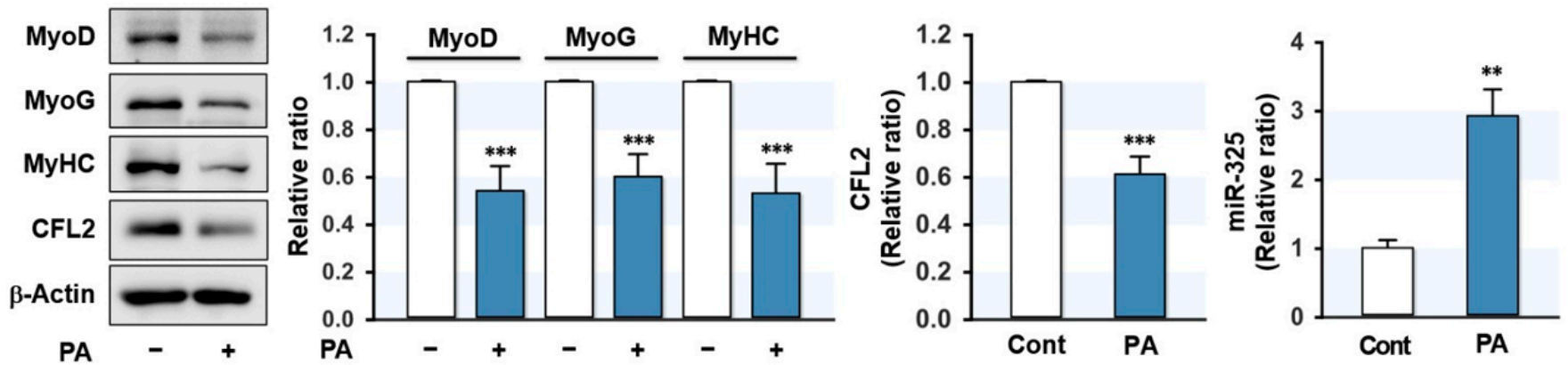

Figure 1. PA inhibited myoblast differentiation but enhanced miR-325-3p expression. (A) C2C12 myoblasts were pretreated with BSA-vehicle (Cont) or PA $(100 \mu \mathrm{M})$ for $24 \mathrm{~h}$ and induced to differentiate for five days. Cells were subjected to immunocytochemistry with MyHC antibody (green) and Hoechst 33342 (blue) to verify differentiation. Scale bar: $50 \mu \mathrm{m}$. (B) Quantitative analysis of differentiation index, fusion index, and MyHC-positive area. (C,D) After pretreatment with PA, cells were differentiated for three days and immunoblotted with antibodies for myogenic factors (MyoD, MyoG, and MyHC) and CFL2. Intensities were normalized versus $\beta$-actin. (E) The expressions of miR-325-3p were determined by $q$ RT-PCR and normalized versus U6. Immunoblot and $q$ RT-PCR results are shown as relative ratios versus control. All results are presented as the means \pm SEMs $(n>3)$, and levels of significance are presented as ${ }^{* *}, p<0.01 ;{ }^{* *}, p<0.001$ vs. controls.

\subsection{MiR-325-3p Directly Targeted CFL2 3'UTR}

Since miR-325-3p and CFL2 levels appeared to be inversely related in myoblasts, we next examined whether miR-325-3p directly targets and downregulates CFL2 expression. In silico analysis using TargetScan suggested that the $3^{\prime}$ UTR of CFL2 mRNA possesses a tentative binding site for the miR-325-3p seed sequence (Figure 2A). To investigate direct interaction between miR-325-3p and the CFL2 $3^{\prime} \mathrm{UTR}$, we constructed a luciferase reporter pmirGLO vector containing a CFL2 3'UTR segment of wild-type (CFL2wt) or mutant binding site (CFL2mut) for miR-325-3p (Figure 2B), and then co-transfected with miR-325-3p mimic or scRNA into C2C12 cells. As shown in Figure $2 \mathrm{C}$, transfection of miR-325-3p mimic effectively reduced the luciferase activity of the wild-type (CFL2wt), whereas a mutant construct in the miR-325-3p binding site (CFL2mut) completely abolished the effect of miR-325-3p mimic on the luciferase activity of CFL2wt. Since direct binding between miR-325-3p and the 3'UTR of CFL2 was confirmed by luciferase reporter analysis, we considered miR-325-3p induction might inhibit the protein level of CFL2 in myoblasts. To investigate this, we transfected C2C12 cells with scRNA or miR-325-3p mimic and then analyzed CFL2 protein and mRNA expressions. Transfection of miR-325-3p mimic decreased CFL2 protein significantly compared with scRNA transfection (Figure 2D). In addition, CFL2 mRNA level was also decreased by miR-325-3p mimic as determined by RTPCR and $q$ RT-PCR (Figure 2E), indicating that miR-325-3p downregulated CFL2 expression by directly binding to the $3^{\prime}$ UTR of CFL2. 
A

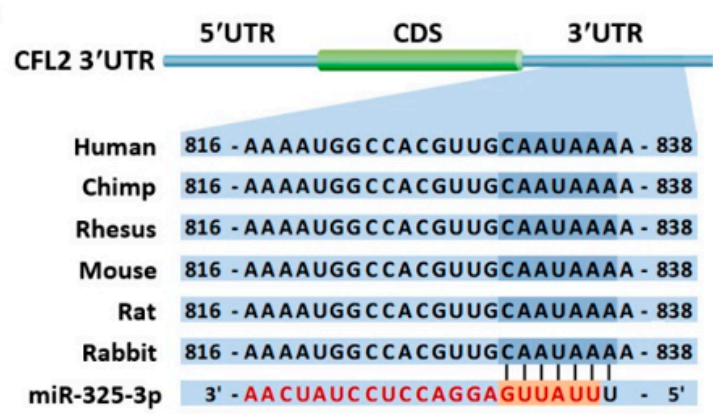

B

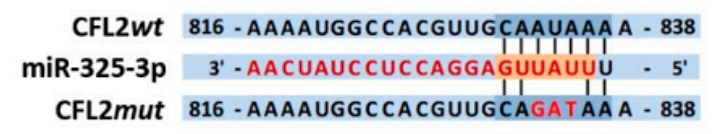

C

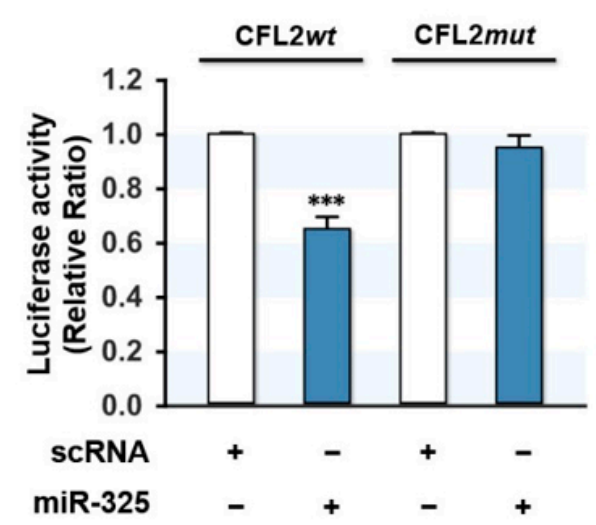

D
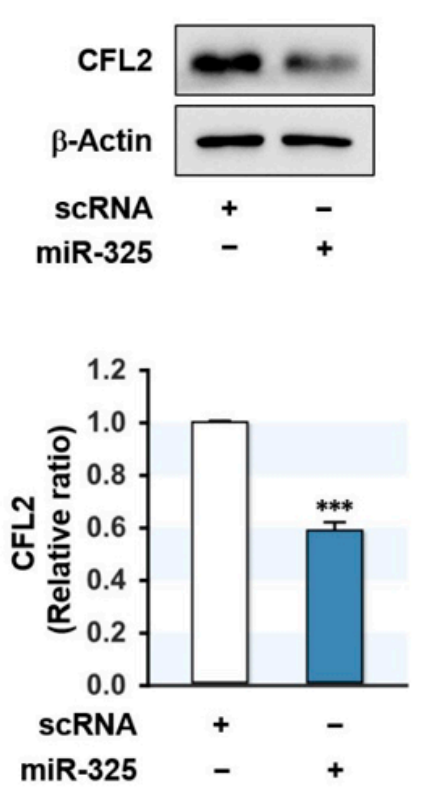

E

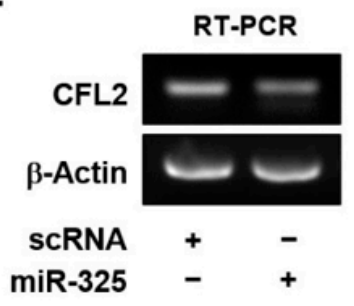

Figure 2. MiR-325-3p regulated CFL2 expression by binding to the 3'UTR of CFL2. (A) Putative binding sites of miR-325-3p on the 3'UTR fragments of CFL2 mRNA. (B) Sequence alignment of miR-325-3p binding site with wild-type (CFL2wt) or mutant (CFL2mut) 3'UTR of CFL2. (C) MiR-325-3p mimic or scrambled control RNA (scRNA) were co-transfected with a dual-luciferase reporter construct containing CFL2wt or CFL2mut in C2C12 cells, and relative luciferase activity was measured $24 \mathrm{~h}$ after transfection. (D) CFL2 protein levels were analyzed $24 \mathrm{~h}$ after transfection with $200 \mathrm{nM}$ of scRNA control or miR-325-3p mimic by immunoblotting. (E) The mRNA expressions were determined by RT-PCR (upper panel) and $q$ RT-PCR (lower panel). Immunoblot and $q$ RT-PCR results are shown as relative ratios versus scRNA control. All results are presented as the means \pm SEMs $(n>3)$, and levels of significance are presented as ${ }^{* *}, p<0.01 ;{ }^{* * *}, p<0.001$ vs. scRNA controls.

\subsection{MiR-325-3p Increased F-Actin and Nuclear Yes-Associated Protein (YAP)}

In a previous study, knockdown of CFL2 provoked the accumulation of F-actin in myoblasts [25], and thus, we hypothesized that miR-325-3p increases F-actin by inhibiting CFL2 expression in myoblasts. Transfection of myoblasts with siCFL2 significantly decreased CFL2 level by $\sim 60 \%$ (Figure 3A) and transfection with miR-325-3p mimic efficiently elevated ( $>200$-fold) the cellular level of miR-325-3p in myoblasts (data not shown). Under this experimental condition, miR-325-3p mimic or siCFL2 dramatically increased F-actin as determined with FITC-coupled phalloidin (Figure 3B). Because actin levels remained constant during differentiation regardless of treatments, the induction of F-actin accumulation by miR-325-3p mimic was ascribed to lack of actin depolymerization due to CFL2 suppression. Recently, it was reported that F-actin accumulation inhibits phosphorylation of a transcriptional coactivator YAP and induces the nuclear translocation of YAP, leading to activation of proliferative transcriptional programs in the Hippo signaling pathway [31,32]. In the present study, transfection with miR-325-3p mimic decreased the 
phosphorylation of YAP (pYAP) in the cytosol and redistributed YAP to the nucleus from the cytosol (Figure 3C,D), implying that the effects of miR-325-3p on F-actin and YAP might stimulate the proliferation of $\mathrm{C} 2 \mathrm{C} 12$ myoblasts.

A
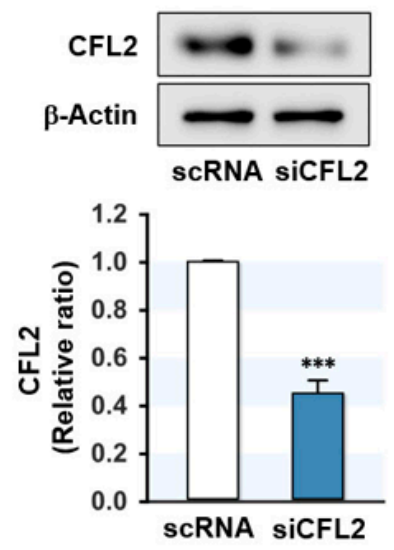

C

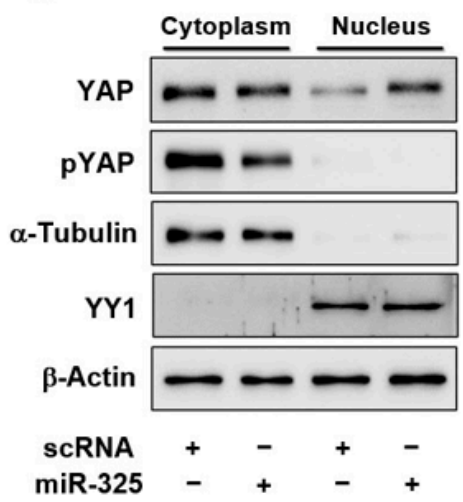

B

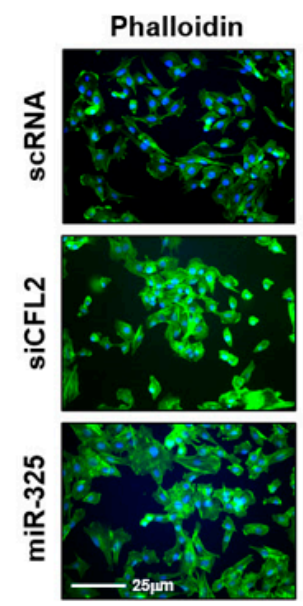

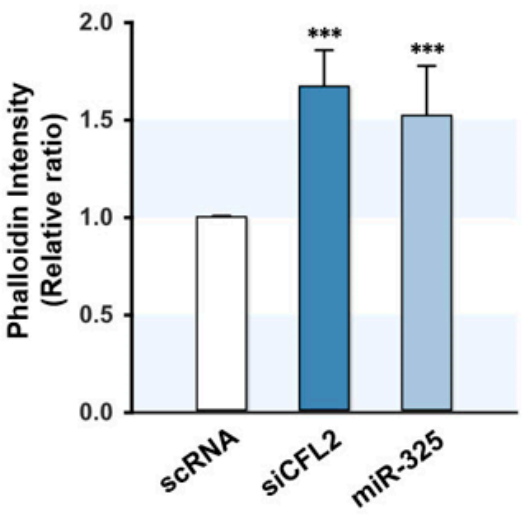

D

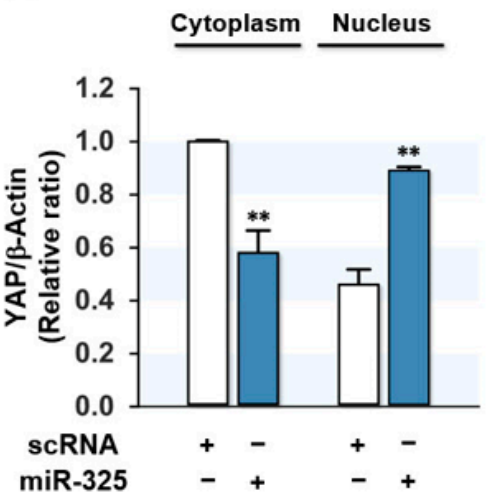

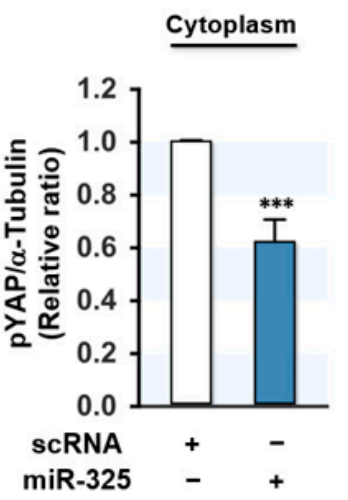

Figure 3. MiR-325-3p increased F-actin and nuclear YAP levels. (A) C2C12 myoblasts were transfected with $200 \mathrm{nM}$ of scRNA or CFL2 siRNA (siCFL2), and CFL2 protein expression was determined $24 \mathrm{~h}$ after transfection by immunoblotting. Intensities were normalized versus $\beta$-actin. (B) Representative images of FITC-phalloidin (green) and Hoechst 33342 (blue) staining after $24 \mathrm{~h}$ of transfection. Scale bar: $25 \mu \mathrm{m}$. Phalloidin intensities were analyzed by ImageJ software. (C,D) YAP and phosphorylated YAP (pYAP) protein expressions in the nuclear and cytoplasmic fractions were determined by immunoblotting after $24 \mathrm{~h}$ of transfection with scRNA or miR-325-3p mimic into C2C12 myoblasts. The quality of subcellular fractionation was confirmed using cytoplasmic ( $\alpha$-Tubulin) or nuclear (YY1) markers. Immunoblot results are shown as relative ratios versus scRNA control. All results are presented as the means \pm SEMs $(n>3)$, and levels of significance are presented as ${ }^{* *}, p<0.01 ;{ }^{* *}, p<0.001$ vs. scRNA controls.

\subsection{MiR-325-3p Promoted Myoblast Proliferation}

To analyze the effect of miR-325-3p on myoblast proliferation, we determined the EdU incorporation in myoblasts after $24 \mathrm{~h}$ of siCFL2 or miR-325-3p mimic transfection. Consistent with our previous finding, knockdown of CFL2 by siCFL2 dramatically increased the percentage of EdU-positive cells as compared with scRNA control (Figure 4A,B), indicating increased myoblast proliferation. Interestingly, transfection with miR-325-3p mimic also significantly increased the percentage of EdU-positive myoblasts, whereas co-transfection with antimiR-325-3p completely abolished the increased EdU incorporation by miR-325-3p mimic in myoblasts (Figure 4A,B), suggesting that miR-325-3p mimic promoted myoblast proliferation. Next, we investigated the expressions of genes related to cell proliferation and cell cycle progression, such as proliferating cell nuclear antigen (PCNA) and CCND1, by $q$ RT-PCR. Expectedly, PCNA and CCND1 were significantly upregulated in myoblasts 
transfected with miR-325-3p mimic (Figure 4C). In addition, the effect of miR-325-3p on the cell cycle was assessed by fluorescence-activated cell sorting. As shown in Figure 4D, miR-325-3p mimic transfection lowered the proportion of cells in the G0/G1 phase while increased in the $S$ and G2/M phase, suggesting that miR-325-3p stimulated cell cycle progression. Collectively, miR-325-3p mimic promoted myoblast proliferation and cell cycle progression.

A

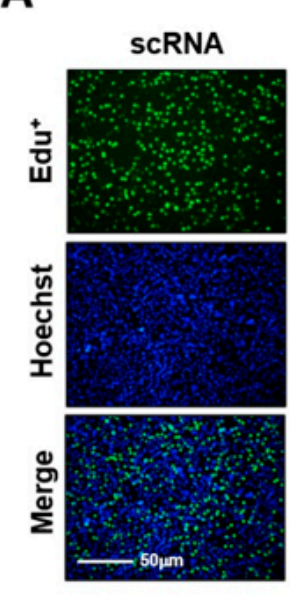

C

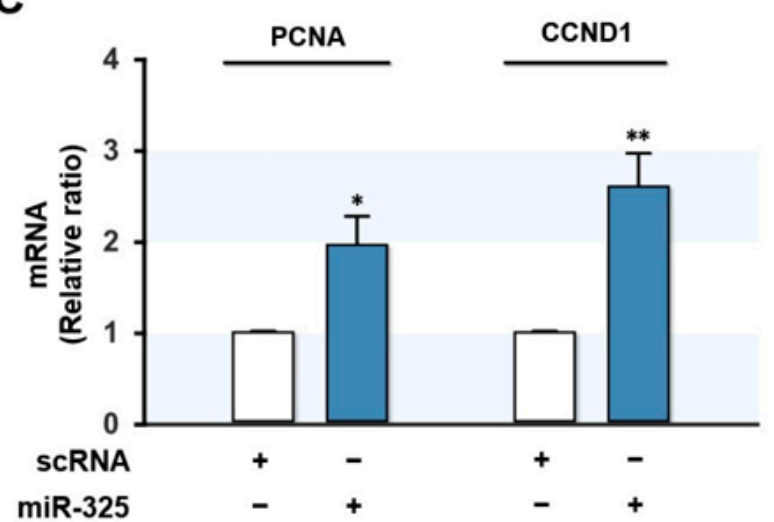

B

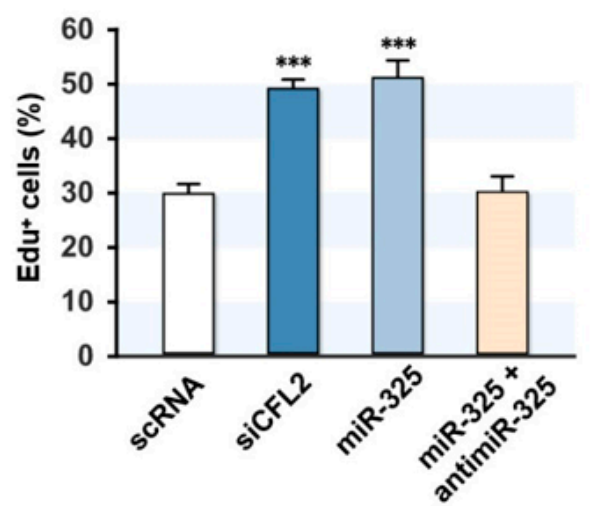

D

Figure 4. MiR-325-3p promoted myoblast proliferation and cell cycle progression. C2C12 cells were transfected with $200 \mathrm{nM}$ of scRNA, siCFL2, miR-325-3p mimic (miR-325), or antimiR-325-3p (antimiR-325). (A) EdU assays were conducted $24 \mathrm{~h}$ after transfection. Cells undergoing DNA replication were labeled with EdU (green) and nuclei with Hoechst 33342 (blue). Scale bar: $50 \mu \mathrm{m}$. (B) Percentages of EdU-positive cells were determined by the ImageJ program. (C) PCNA and CCND1 levels in C2C12 myoblasts were determined by $q$ RT-PCR and normalized with U6. (D) Flow cytometry after $24 \mathrm{~h}$ of transfection with scRNA or miR-325-3p mimic. All results are presented as the means \pm SEMs $(n>3)$, and levels of significance are presented as ${ }^{*}, p<0.05 ;{ }^{* *}, p<0.01 ; * * *, p<0.001$ vs. scRNA controls.

\subsection{MiR-325-3p Suppressed the Expressions of Myogenic Factors}

Cell cycle exit and proliferation arrest have long been recognized as necessary conditions for progenitor cell differentiation $[2,33]$. Therefore, we investigated whether miR325-3p influences the expressions of myogenic factors. C2C12 cells were transfected with scRNA, siCFL2, miR-325-3p mimic, or antimiR-320a-3p, and the protein expressions of MyoD, MyoG, and MyHC were assessed on differentiation day 3 (Figure 5A,B). As was expected, siCFL2 reduced the level of CFL2 by $~ 50 \%$ versus the scRNA control and significantly reduced the expressions of the myogenic factors MyoD, MyoG, and MyHC. Interestingly, transfection with miR-325-3p mimic also suppressed CFL2 level markedly and reduced the expressions of $\mathrm{MyoD}, \mathrm{MyoG}$, and $\mathrm{MyHC}$ versus scRNA controls, which 
suggested that CFL2 inhibition or miR-325-3p mimic are causally linked to the suppressions of myogenic factors. Furthermore, co-transfection with antimiR-325-3p almost completely abrogated the inhibitory effect of miR-325-3p mimic on the expressions of myogenic factors (Figure 5A,B). These results demonstrated that miR-325-3p suppresses myogenic factors expressions in $\mathrm{C} 2 \mathrm{C} 12$ myoblasts.

A

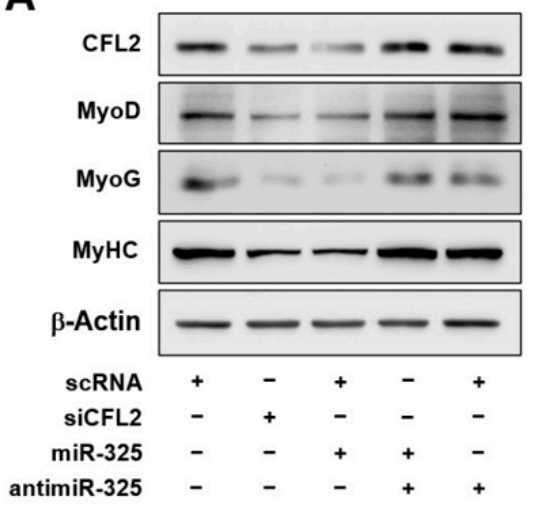

B
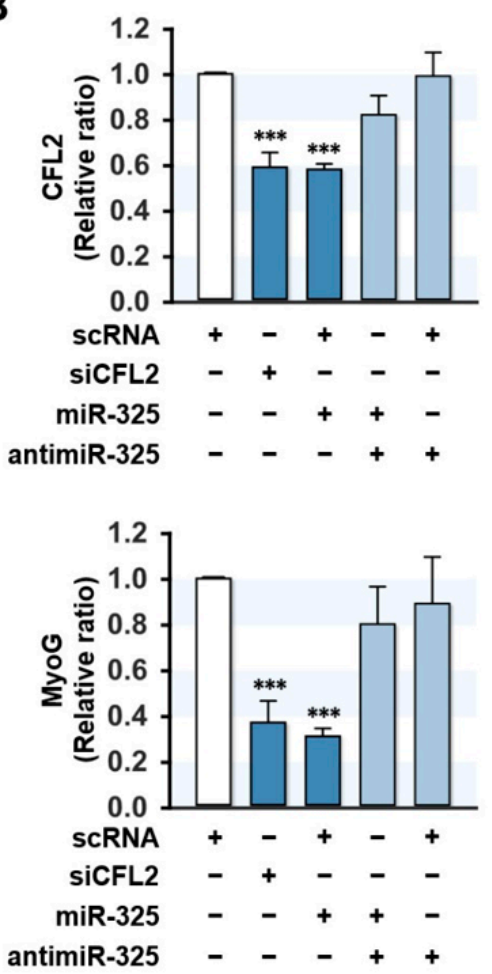
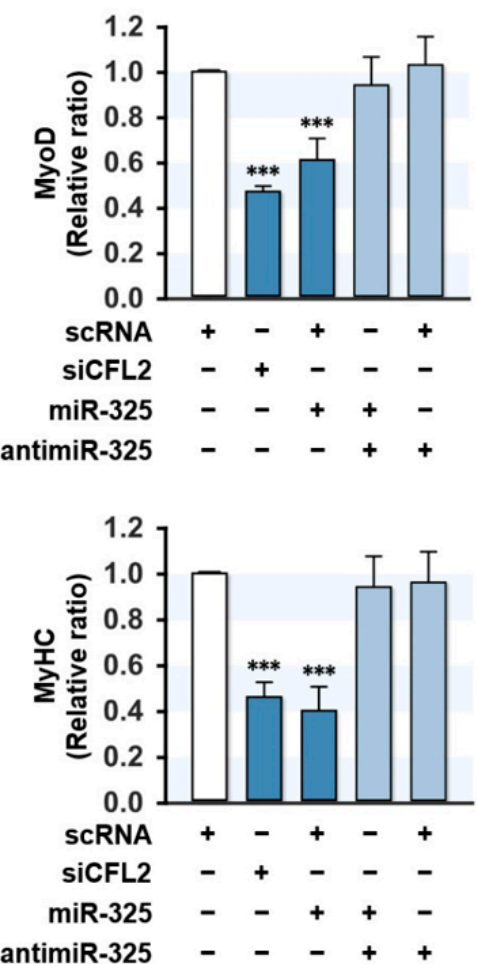

Figure 5. MiR-325-3p suppressed myogenic factors expressions. C2C12 cells were transfected with 200 nM of scRNA, siCFL2, miR-325-3p mimic (miR-325), or antimiR-325-3p (antimiR-325) and differentiated for three days. (A) Representative immunoblots of CFL2, MyoD, MyoG, and MyHC levels. (B) Quantitative analysis of immunoblots with antibodies for myogenic factors (MyoD, MyoG, and MyHC) and CFL2. Intensities were normalized versus $\beta$-actin. All results are presented as the means \pm SEMs $(n>3)$, and levels of significance are presented as ${ }^{* *}, p<0.001$ vs. scRNA controls.

\subsection{MiR-325-3p Impeded Myogenic Differentiation}

The suppressive effect of miR-325-3p mimic on myogenic factors suggests miR-325-3p might impair myoblast differentiation and myotube formation. Therefore, we transfected C2C12 cells with scRNA, siCFL2, miR-325-3p mimic, or antimiR-325-3p and allowed them to differentiate for five days (Figure 6). Immunocytochemistry with an MyHC antibody was used to analyze myoblast differentiation, followed by quantitative image analysis. CFL2 knockdown by siCFL2 significantly reduced myogenic differentiation, fusion, and myotube formation in myoblasts as determined by differentiation index, fusion index, percentage areas of MyHC-positive cells, and myotube widths, suggesting that CFL2 depletion impaired myoblast differentiation (Figure 6A,B). Similarly, transfection with miR325-3p mimic also significantly decreased myoblast differentiation and myotube formation as assessed by immunocytochemistry and image analysis (Figure 6A,B). Additionally, co-transfection with antimiR-325-3p abolished the inhibitory effect of miR-325-3p mimic on myoblast differentiation (Figure 6A,B). Thus, these results suggest that miR-325-3p impaired myogenic differentiation and myotube formation in C2C12 myoblasts. 
B

A

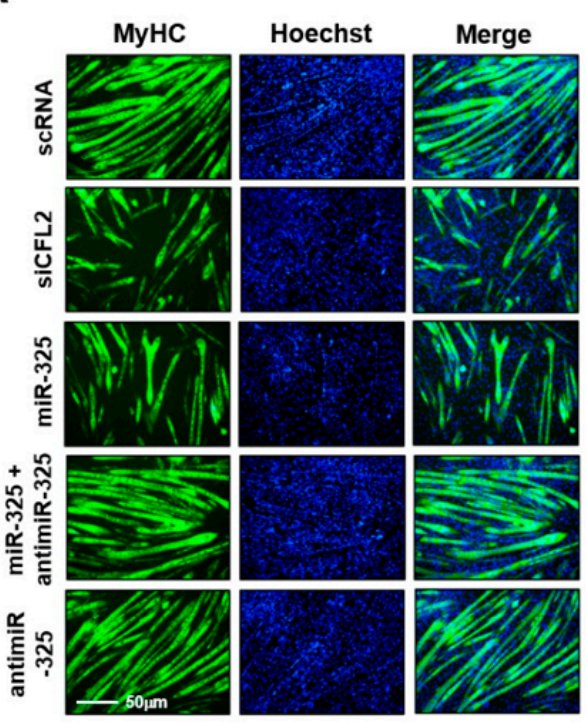

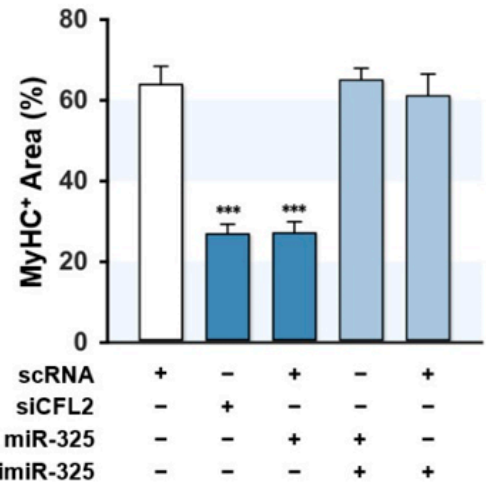

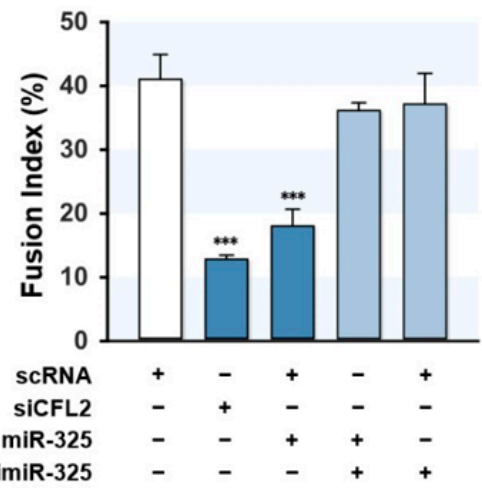

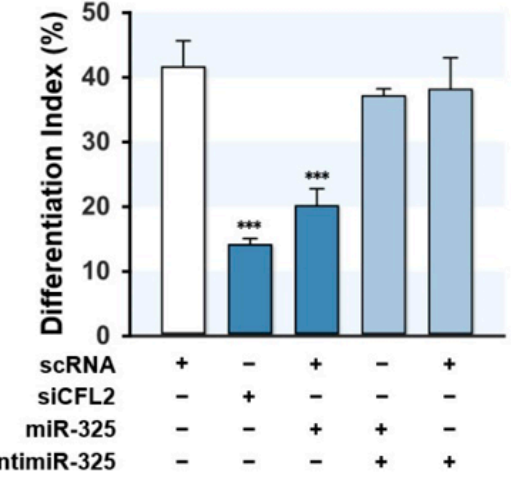

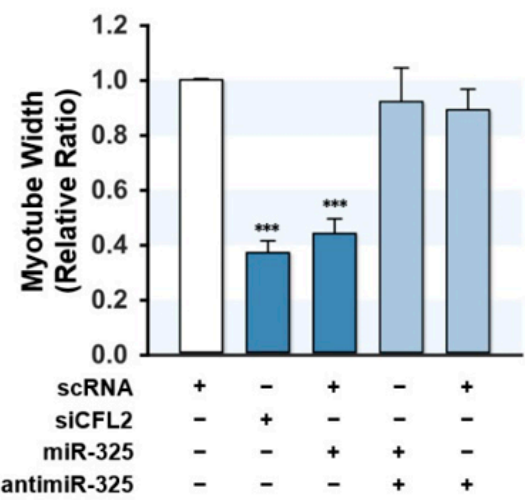

Figure 6. MiR-325-3p negatively regulated myogenic differentiation and myotube formation. C2C12 cells were transfected with $200 \mathrm{nM}$ of scRNA, siCFL2, miR-325-3p mimic (miR-325), or antimiR-325-3p (antimiR-325) and differentiated for five days. (A) Representative immunocytochemistry with MyHC antibody (green) and Hoechst 33342 (blue). Scale bar: $50 \mu \mathrm{m}$. (B) Quantitative analysis of MyHC-positive area, differentiation index, fusion index, and myotube width. All results are presented as the means \pm SEMs $(n>3)$, and levels of significance are presented as ${ }^{* * *}, p<0.001$ vs. scRNA controls.

\section{Discussion}

Although miRNAs are known to play a significant role in myogenesis and to be involved in the pathogenesis of muscle wasting $[9,10,16]$, the molecular mechanisms whereby SFA or obesity-induced miRNAs affect myogenesis remain poorly understood. Here, we unveiled an essential role of miR-325-3p on CFL2 expression, myoblast proliferation, and myogenic differentiation, which contributes to our current understanding of the miRNAmediated myogenic regulatory mechanism in the background of obesity. We summarize our key findings as follows: (i) PA impaired the differentiation of myoblasts, downregulated CFL2, and upregulated miR-325-3p expression; (ii) MiR-325-3p directly targeted the $3^{\prime}$ UTR of CFL2 and suppressed CFL2 expression; (iii) transfection of C2C12 myoblasts with miR-325-3p mimic promoted actin polymerization and induced the nuclear accumulation of YAP; (iv) MiR-325-3p mimic also enhanced myoblast proliferation and cell cycle progression; (v) MiR-325-3p mimic reduced the expressions of myogenic factors and inhibited myogenic differentiation. Therefore, these results indicate that miR-325-3p plays a vital role in myogenesis by regulating the expression of CFL2 and suggest miRNA-mediated myogenic regulation in association with SFA and obesity.

Our results in the current study demonstrate the regulation of myogenesis by miR-325$3 p$ and support our hypothesis that certain miRNAs induced by SFA impair myogenesis. Notably, miR-325-3p markedly upregulated by PA promoted myoblast proliferation and cell cycle progression. Since it has been known that myoblast proliferation and myogenic differentiation are inversely related during myogenesis, proliferation arrest is a prerequisite for the differentiation of myoblasts $[2,33]$. In this regard, the inhibition of myogenic differentiation by miR-325-3p is primarily attributed to the promotion of cell cycle pro- 
gression and proliferation in myoblasts. Interestingly, the upregulation of miR-325-3p has been implicated in the occurrence and progression of various malignancies [34-37], and miR-325-3p overexpression promoted cancer cell proliferation, invasion, and metastasis [34]. Although several other studies showed the suppressive effect on proliferation by miR-325-3p in cancer cells [38-40], this discrepancy regarding the effect of miR-325-3p on cell proliferation might be explained by the cell type-dependent differences in composition of protein components, target proteins abundance, and miR-325-3p level. In this respect, it is worth noting that CFL2 as a target of miR-325-3p is a skeletal muscle-specific protein that is upregulated in myoblasts during myogenic differentiation $[19,25]$.

Then, what mechanism is responsible for miR-325-3p-induced myoblast proliferation and cell cycle progression? According to one of the important findings of the present study, miR-325-3p promoted F-actin formation by directly inhibiting the expression of CFL2 (Figure 3). CFL2 has been recognized as a necessary component of actin remodeling due to its ability to sever F-actin, which regulates mechanical stress in the cytoskeleton [20,24]. The actin cytoskeleton dynamics has been suggested to be a critical regulator of YAP in the Hippo signaling pathway [41], which controls tissue and organ sizes in animals by modulating cell proliferation and differentiation [42]. The nuclear translocations of cytosolic YAP and TAZ activate proliferative and anti-apoptotic transcriptional activities in this pathway [43]. Furthermore, F-actin accumulation was shown to diminish the phosphorylation of YAP/TAZ and, consequently, increases their nuclear translocation and cell proliferation [31,32]. In this regard, F-actin severing proteins such as CFL and Gelosin act as negative regulators of YAP by increasing its phosphorylation and degradation $[23,44]$. Accordingly, actin remodeling mediated by CFL is directly connected to the regulation of cell proliferation via the nuclear translocation of YAP $[23,24]$. In a previous study, we found knockdown of CFL2 resulted in F-actin accumulation and increased cell cycle progression and cell proliferation in $\mathrm{C} 2 \mathrm{C} 12$ myoblasts [25]. Torrini et al. also discovered that CFL2 depletion enhanced F-actin levels and activated YAP in cardiomyocytes [45]. Moreover, cytochalasin D, a potent actin depolymerizer, inhibited the nuclear translocation of YAP, whereas jasplakinolide, an F-actin inducer, promoted its nuclear translocation [45]. Our data suggest that the stimulatory effect of miR-325-3p on cell proliferation is primarily related to the disruption of actin dynamics caused by CFL2 suppression. Collectively, miR-325-3p inhibited CFL2 expression, elevated F-actin accumulation, induced the nuclear translocation of YAP, and ultimately led to myoblast proliferation and delayed myogenic differentiation.

Although the regulatory mechanism responsible for miR-325-3p induction by PA was not investigated in this work, we speculate that specific transcription factors activated by PA or obesity may mediate the upregulation of miR-325-3p in myoblasts. To address this issue, we analyzed the promoter regions of human and mouse miR-325-3p and found an optimal consensus binding site for the E2F1 transcription factor. E2F1, a member of the E2F family of transcription factors, has often been implicated in metabolic regulation and acts as a pivotal player in the cell cycle progression for cell growth and survival [46]. Previously, Bo et al. showed E2F1 bound to miR-325-3p promoter and enhanced miR-325-3p expression in cardiomyocytes, and E2F1 knockout mice exhibited a low miR-325-3p level, indicating that E2F1 is a transcriptional activator of miR-325-3p [47]. Interestingly, E2F1 levels were elevated in the adipose tissue of obese humans [48] and obese mouse models, such as high-fat diet (HFD)-fed mice and ob/ob mice [49]. Given the functions and regulation of E2F1 in proliferation and metabolism, it appears that E2F1 might play a critical role in the upregulation of miR-325-3p in obesity. Another interesting recent study demonstrated that cellular treatment of transforming growth factor- $\beta$ (TGF- $\beta$ ) increased miR-325-3p expression in colorectal carcinoma cells [35]. TGF- $\beta$ is a well-known key modulator of insulin resistance in metabolic disorders associated with obesity [50]. Indeed, circulating TGF- $\beta$ levels were increased in obese humans, ob/ob mice, and HFD-induced obese mice [51]. Although further study is warranted, the results of previous studies suggest 
that the activation of E2F1 or TGF- $\beta$ in a background of obesity may induce miR-325-3p expression, thereby provoking impaired myogenesis and muscle wasting.

\section{Conclusions}

This study demonstrates that miR-325-3p plays an essential role in actin remodeling and myogenic differentiation in $\mathrm{C} 2 \mathrm{C} 12$ myoblasts. PA inhibited differentiation of myoblasts and induced miR-325-3p expression. Interestingly, miR-325-3p inhibited the expression of CFL2, which is required for myogenic differentiation, via directly targeting the $3^{\prime} U T R$ of CFL2 mRNA. Transfection of miR-325-3p mimic increased F-actin and stimulated the nuclear translocation of YAP, thus promoting myoblast proliferation and impaired myogenic differentiation. The roles of miR-325-3p on CFL2 expression and myogenic differentiation suggest a novel miRNA-mediated mechanism that regulates myogenesis in the background of obesity. From a clinical point of view, miR-325-3p may be a vital mediator between obesity and muscle wasting and will provide a means of developing practical diagnostic and therapeutic approaches for muscle wasting and sarcopenic obesity.

Supplementary Materials: The following are available online at https:/ /www.mdpi.com/article/10 $.3390 /$ cells10102725/s1, Supplementary data: Analysis of miR-325-3p expression using an Affymetrix GeneChip miRNA 4.0 array, Table S1: Oligonucleotide sequences for transfection, Table S2: Primer lists and PCR conditions for qRT-PCR, RT-PCR and cloning, Table S3: Antibodies list.

Author Contributions: W.L. and M.T.N. conceived and designed the experiments; M.T.N. performed the experiments and analyzed the data; W.L. wrote the manuscript. All authors have read and agreed to the published version of the manuscript.

Funding: This study was supported by the National Research Foundation of Korea (NRF) funded by the Korean government (Grant no. NRF-2019R1F1A1040858).

Conflicts of Interest: The authors declare no conflict of interest.

\section{References}

1. Dumont, N.A.; Bentzinger, C.F.; Sincennes, M.C.; Rudnicki, M.A. Satellite Cells and Skeletal Muscle Regeneration. Compr. Physiol. 2015, 5, 1027-1059. [CrossRef] [PubMed]

2. Chal, J.; Pourquie, O. Making muscle: Skeletal myogenesis in vivo and in vitro. Development 2017, 144, 2104-2122. [CrossRef] [PubMed]

3. Cruz-Jentoft, A.J.; Sayer, A.A. Sarcopenia. Lancet 2019, 393, 2636-2646. [CrossRef]

4. Sartori, R.; Romanello, V.; Sandri, M. Mechanisms of muscle atrophy and hypertrophy: Implications in health and disease. Nat. Commun. 2021, 12, 330. [CrossRef] [PubMed]

5. Batsis, J.A.; Villareal, D.T. Sarcopenic obesity in older adults: Aetiology, epidemiology and treatment strategies. Nat. Rev. Endocrinol. 2018, 14, 513-537. [CrossRef] [PubMed]

6. Barazzoni, R.; Bischoff, S.; Boirie, Y.; Busetto, L.; Cederholm, T.; Dicker, D.; Toplak, H.; Van Gossum, A.; Yumuk, V.; Vettor, R. Sarcopenic Obesity: Time to Meet the Challenge. Obes. Facts 2018, 11, 294-305. [CrossRef] [PubMed]

7. Akhmedov, D.; Berdeaux, R. The effects of obesity on skeletal muscle regeneration. Front. Physiol. 2013, 4, 371. [CrossRef]

8. Teng, S.; Huang, P. The effect of type 2 diabetes mellitus and obesity on muscle progenitor cell function. Stem. Cell Res. Ther. 2019, 10, 103. [CrossRef]

9. Ji, C.; Guo, X. The clinical potential of circulating microRNAs in obesity. Nat. Rev. Endocrinol. 2019, 15, 731-743. [CrossRef]

10. Ortiz-Dosal, A.; Rodil-Garcia, P.; Salazar-Olivo, L.A. Circulating microRNAs in human obesity: A systematic review. Biomarkers 2019, 24, 499-509. [CrossRef]

11. Krol, J.; Loedige, I.; Filipowicz, W. The widespread regulation of microRNA biogenesis, function and decay. Nat. Rev. Genet. 2010, 11,597-610. [CrossRef]

12. Bartel, D.P. MicroRNAs: Genomics, biogenesis, mechanism, and function. Cell 2004, 116, 281-297. [CrossRef]

13. Saliminejad, K.; Khorram Khorshid, H.R.; Soleymani Fard, S.; Ghaffari, S.H. An overview of microRNAs: Biology, functions, therapeutics, and analysis methods. J. Cell Physiol. 2019, 234, 5451-5465. [CrossRef]

14. Zhao, Y.; Chen, M.; Lian, D.; Li, Y.; Li, Y.; Wang, J.; Deng, S.; Yu, K.; Lian, Z. Non-Coding RNA Regulates the Myogenesis of Skeletal Muscle Satellite Cells, Injury Repair and Diseases. Cells 2019, 8, 988. [CrossRef]

15. Mok, G.F.; Lozano-Velasco, E.; Munsterberg, A. MicroRNAs in skeletal muscle development. Semin. Cell Dev. Biol. 2017, 72, 67-76. [CrossRef]

16. Sannicandro, A.J.; Soriano-Arroquia, A.; Goljanek-Whysall, K. Micro(RNA)-managing muscle wasting. J. Appl. Physiol. 2019, 127, 619-632. [CrossRef] 
17. Guerin, C.M.; Kramer, S.G. Cytoskeletal remodeling during myotube assembly and guidance: Coordinating the actin and microtubule networks. Commun. Integr. Biol. 2009, 2, 452-457. [CrossRef]

18. Heng, Y.W.; Koh, C.G. Actin cytoskeleton dynamics and the cell division cycle. Int. J. Biochem. Cell Biol. 2010, 42, 1622-1633. [CrossRef]

19. Kanellos, G.; Frame, M.C. Cellular functions of the ADF/cofilin family at a glance. J. Cell Sci. 2016, 129, 3211-3218. [CrossRef]

20. Bravo-Cordero, J.J.; Magalhaes, M.A.; Eddy, R.J.; Hodgson, L.; Condeelis, J. Functions of cofilin in cell locomotion and invasion. Nat. Rev. Mol. Cell Biol. 2013, 14, 405-415. [CrossRef]

21. Agrawal, P.B.; Joshi, M.; Savic, T.; Chen, Z.; Beggs, A.H. Normal myofibrillar development followed by progressive sarcomeric disruption with actin accumulations in a mouse $\mathrm{Cfl} 2$ knockout demonstrates requirement of cofilin-2 for muscle maintenance. Hum. Mol. Genet. 2012, 21, 2341-2356. [CrossRef] [PubMed]

22. De Winter, J.M.; Ottenheijm, C.A.C. Sarcomere Dysfunction in Nemaline Myopathy. J. Neuromuscul. Dis. 2017, 4, 99-113. [CrossRef] [PubMed]

23. Aragona, M.; Panciera, T.; Manfrin, A.; Giulitti, S.; Michielin, F.; Elvassore, N.; Dupont, S.; Piccolo, S. A mechanical checkpoint controls multicellular growth through YAP/TAZ regulation by actin-processing factors. Cell 2013, 154, 1047-1059. [CrossRef]

24. Bernstein, B.W.; Bamburg, J.R. ADF/cofilin: A functional node in cell biology. Trends Cell Biol. 2010, 20, 187-195. [CrossRef]

25. Nguyen, M.T.; Min, K.H.; Kim, D.; Park, S.Y.; Lee, W. CFL2 is an essential mediator for myogenic differentiation in C2C12 myoblasts. Biochem. Biophys. Res. Commun. 2020, 533, 710-716. [CrossRef]

26. Nguyen, M.T.; Min, K.H.; Lee, W. MiR-96-5p Induced by Palmitic Acid Suppresses the Myogenic Differentiation of C2C12 Myoblasts by Targeting FHL1. Int. J. Mol. Sci. 2020, 21, 9445. [CrossRef] [PubMed]

27. Yang, W.M.; Jeong, H.J.; Park, S.W.; Lee, W. Obesity-induced miR-15b is linked causally to the development of insulin resistance through the repression of the insulin receptor in hepatocytes. Mol. Nutr. Food Res. 2015, 59, 2303-2314. [CrossRef] [PubMed]

28. Ryu, H.S.; Park, S.Y.; Ma, D.; Zhang, J.; Lee, W. The induction of microRNA targeting IRS-1 is involved in the development of insulin resistance under conditions of mitochondrial dysfunction in hepatocytes. PLoS ONE 2011, 6, e17343. [CrossRef]

29. Tang, Z.; Yang, Y.; Wang, Z.; Zhao, S.; Mu, Y.; Li, K. Integrated analysis of miRNA and mRNA paired expression profiling of prenatal skeletal muscle development in three genotype pigs. Sci. Rep. 2015, 5, 15544. [CrossRef]

30. Van Pelt, D.W.; Vechetti, I.J., Jr.; Lawrence, M.M.; Van Pelt, K.L.; Patel, P.; Miller, B.F.; Butterfield, T.A.; Dupont-Versteegden, E.E. Serum extracellular vesicle miR-203a-3p content is associated with skeletal muscle mass and protein turnover during disuse atrophy and regrowth. Am. J. Physiol. Cell Physiol. 2020, 319, C419-C431. [CrossRef]

31. Dupont, S. Role of YAP/TAZ in cell-matrix adhesion-mediated signalling and mechanotransduction. Exp. Cell Res. 2016, 343, 42-53. [CrossRef]

32. Dupont, S.; Morsut, L.; Aragona, M.; Enzo, E.; Giulitti, S.; Cordenonsi, M.; Zanconato, F.; Le Digabel, J.; Forcato, M.; Bicciato, S.; et al. Role of YAP/TAZ in mechanotransduction. Nature 2011, 474, 179-183. [CrossRef]

33. Bentzinger, C.F.; Wang, Y.X.; Rudnicki, M.A. Building muscle: Molecular regulation of myogenesis. Cold Spring Harb. Perspect Biol. 2012, 4, a008342. [CrossRef]

34. Wang, H.; Hu, X.; Yang, F.; Xiao, H. MiR-325-3p promotes the proliferation, invasion and EMT of breast cancer cells by directly targeting S100A2. Oncol. Res. Featur. Preclin. Clin. Cancer Ther. 2021, 28, 731-744. [CrossRef]

35. Song, C.; Wang, X.; Zhao, X.; Ai, J.; Qi, Y.; Chen, A. MicroRNA-325-3p contributes to colorectal carcinoma by targeting cytokeratin 18. Oncol. Lett. 2021, 21, 248. [CrossRef]

36. Deng, Q.; Hu, H.; Yu, X.; Liu, S.; Wang, L.; Chen, W.; Zhang, C.; Zeng, Z.; Cao, Y.; Xu-Monette, Z.Y.; et al. Tissue-specific microRNA expression alters cancer susceptibility conferred by a TP53 noncoding variant. Nat. Commun. 2019, 10, 5061. [CrossRef]

37. Braicu, O.L.; Budisan, L.; Buiga, R.; Jurj, A.; Achimas-Cadariu, P.; Pop, L.A.; Braicu, C.; Irimie, A.; Berindan-Neagoe, I. MiRNA expression profiling in formalin-fixed paraffin-embedded endometriosis and ovarian cancer samples. Onco Targets Ther. 2017, 10, 4225-4238. [CrossRef]

38. He, C.; Wang, X.; Du, M.; Dong, Y. LncRNA MSC-AS1 Promotes Colorectal Cancer Progression by Regulating miR-325/TRIM14 Axis. J. Oncol. 2021, 2021, 9954214. [CrossRef]

39. Wang, F.; Wang, F.; Zhang, S.; Xu, X. MicroRNA-325 inhibits the proliferation and induces the apoptosis of T cell acute lymphoblastic leukemia cells in a BAG2-dependent manner. Exp. Ther. Med. 2021, 21, 631. [CrossRef]

40. Li, L.; Ji, Y.; Chen, Y.C.; Zhen, Z.J. MiR-325-3p mediate the CXCL17/CXCR8 axis to regulate angiogenesis in hepatocellular carcinoma. Cytokine 2021, 141, 155436. [CrossRef]

41. Mendez, M.G.; Janmey, P.A. Transcription factor regulation by mechanical stress. Int. J. Biochem. Cell Biol. 2012, 44, 728-732. [CrossRef] [PubMed]

42. Boopathy, G.T.K.; Hong, W. Role of Hippo Pathway-YAP/TAZ Signaling in Angiogenesis. Front. Cell Dev. Biol. 2019, 7, 49. [CrossRef] [PubMed]

43. Zou, R.; Xu, Y.; Feng, Y.; Shen, M.; Yuan, F.; Yuan, Y. YAP nuclear-cytoplasmic translocation is regulated by mechanical signaling, protein modification, and metabolism. Cell Biol. Int. 2020, 44, 1416-1425. [CrossRef] [PubMed]

44. Kim, J.; Jo, H.; Hong, H.; Kim, M.H.; Kim, J.M.; Lee, J.K.; Heo, W.D.; Kim, J. Actin remodelling factors control ciliogenesis by regulating YAP/TAZ activity and vesicle trafficking. Nat. Commun. 2015, 6, 6781. [CrossRef] 
45. Torrini, C.; Cubero, R.J.; Dirkx, E.; Braga, L.; Ali, H.; Prosdocimo, G.; Gutierrez, M.I.; Collesi, C.; Licastro, D.; Zentilin, L.; et al. Common Regulatory Pathways Mediate Activity of MicroRNAs Inducing Cardiomyocyte Proliferation. Cell Rep. 2019, 27, 2759-2771.e2755. [CrossRef]

46. Denechaud, P.D.; Fajas, L.; Giralt, A. E2F1, a Novel Regulator of Metabolism. Front. Endocrinol. 2017, 8, 311. [CrossRef]

47. Bo, L.; Su-Ling, D.; Fang, L.; Lu-Yu, Z.; Tao, A.; Stefan, D.; Kun, W.; Pei-Feng, L. Autophagic program is regulated by miR-325. Cell Death Differ. 2014, 21, 967-977. [CrossRef]

48. Haim, Y.; Blüher, M.; Slutsky, N.; Goldstein, N.; Klöting, N.; Harman-Boehm, I.; Kirshtein, B.; Ginsberg, D.; Gericke, M.; Guiu Jurado, E.; et al. Elevated autophagy gene expression in adipose tissue of obese humans: A potential non-cell-cycle-dependent function of E2F1. Autophagy 2015, 11, 2074-2088. [CrossRef]

49. Choi, Y.; Jang, S.; Choi, M.-S.; Ryoo, Z.Y.; Park, T. Increased expression of FGF1-mediated signaling molecules in adipose tissue of obese mice. J. Physiol. Biochem. 2016, 72, 157-167. [CrossRef]

50. Pervin, S.; Singh, V.; Tucker, A.; Collazo, J.; Singh, R. Modulation of transforming growth factor- $\beta$ / follistatin signaling and white adipose browning: Therapeutic implications for obesity related disorders. Horm. Mol. Biol. Clin. Investig. 2017, 31. [CrossRef]

51. Yadav, H.; Quijano, C.; Kamaraju, A.K.; Gavrilova, O.; Malek, R.; Chen, W.; Zerfas, P.; Zhigang, D.; Wright, E.C.; Stuelten, C.; et al. Protection from Obesity and Diabetes by Blockade of TGF- $\beta /$ Smad3 Signaling. Cell Metab. 2011, 14, 67-79. [CrossRef] 